\title{
Zinc Improves the Bone Mechanical Strength in Ovariectomized Rat Model by Restoring Bone Composition and Hydroxyapatite Crystallite Dimension
}

\author{
Payal Bhardwaj ${ }^{1 *}$, DurgVijay Rai ${ }^{1,2}$ and Mohan Lal Garg \\ ${ }^{1}$ Department of Biophysics, Panjab University, Chandigarh, India \\ ${ }^{2}$ Faculty of Biomedical Engineering, Shobhit University, Meerut, Uttar Pradesh, India
}

\begin{abstract}
Purpose: Osteoporosis is a bone metabolic disorder which is well known to increase bone porosity and is the outcome of various factors like ageing, genetic, nutritional deficiency, decreased calcium uptake, and last but the most important hormonal imbalances. Hormonal imbalance is one of the major factors affecting women worldwide and leading to osteoporosis. Trace elements play a very essential role in number of pathological conditions. Ingestion of zinc in the early stages of bone loss may be more beneficial in mitigating bone loss and also in improving the overall strength of the bone. In the current work, we have intended to extract the information pertaining to the mechanical strength of bone, bone tissue composition and hydroxyapatite crystallite size upon supplementing zinc in the osteopenic condition.
\end{abstract}

Methods: Forty eight wistar female rats in two set of twenty four animals each were assigned to four groups: Control, Zinc, Ovariectomized (OVX) and OVX+Zinc. Duration of the treatment period was of eight weeks. Biochemical estimations were carried out to make comparison between the treatment groups based on bone metabolism markers in serum. Bone mechanical strength of both the bones i.e., femur and tibia, was assessed using texture analyzer. Also, bone matrix analysis using Fourier transformer infrared spectroscopy and X-ray diffraction studies were carried out for all the treatment groups.

Results: Estradiol levels decreased and tartarate-resistant acid phosphatase $5 \mathrm{~b}$ levels increased in the OVX group. Zinc supplemented following ovariectomy regulated these levels. The OVX group showed significantly higher serum alkaline phosphatase levels, which recovered upon zinc supplementation. Further, zinc plays a potential role in preventing bone tissue deterioration by restoring its composition and microstructure in the post-menopausal condition, thereby, maintaining the mechanical strength of the bone.

Conclusion: These findings suggested that alterations in the bone tissue material properties following estrogen deficiency can be averted by zinc if administered at early stages of bone loss.

Keywords: Estrogen; Zinc; Rat bone; Bone composition; Crystallite size; Texture analyzer

Abbreviations: OVX: Ovariectomized; ROS: Reactive oxygen species; RANKL: Receptor activator for nuclear factor $\mathrm{kB}$ ligand; FDA: Food and Drug Administration; TRAP: Tartarate resistant acid phosphatase; ALP: Alkaline phosphatase; PNPP: p-Nitro phenyl phosphate; FTIR: Fourier transformer infrared spectroscopy; $\mathrm{KBr}$ : Potassium Bromide; SD: Standard deviation; OPG: Osteoprotegrin; XRD: X-Ray Diffraction.

\section{Introduction}

Osteoporosis is one of the most common bone metabolic disorders that cause degradation of bones mechanical function as a result of changes in its material properties [1]. The alterations in the bone composition and structure occur as a result of changes in metabolic conditions such as hormonal changes, steroids, dietary intake, and lifestyle [2,3]. Postmenopausal condition is the most common type of hormonal imbalances that is prevailing amongst women. The clinical importance of osteoporosis as a result of estrogen deficiency has been extensively investigated in current years because of the large number of people getting affected. The factors that contribute to bone strength include two parameters bone quantity and quality. Quantity is measured in terms of bone mineral density and bone quality includes arrangement or micro architecture of both the collagen and hydroxyapatite, and cellular activity [4]. Large number of therapies for osteoporosis is available these days that fall into two categories: antiresorptive drugs, and anabolic drugs. However, these therapies encompass many adverse effects like malignant tumor formation with hormone therapy and gastrointestinal tolerance problems with bisphosphonates, which may exclude their long-term administration $[5,6]$. Thus, there is a need for an alternative therapy that can improve bone health without inducing adverse effects. Nutrition supplements such as vitamins [7], proteins [8] and amino acids [9] are well acknowledged to promote bone remodeling process and to augment mineral absorption.

Insufficient amount of micronutrients and macronutrients have a strong implication in the pathogenesis of number of metabolic disorders. Nutritional factors carry an important role in delaying the metabolic bone disorders. Numbers of evidences are present in the literature showing the role of food factors in regulating bone homeostasis [1012]. Intake of dietary supplements can have restorative effects on the bone loss and therefore in osteoporosis prevention. A trace element is one such dietary element which is required for the proper functioning of the physiological system. Out of all the trace elements known, zinc plays a very essential role in the maintenance of skeletal system. Many

*Corresponding author: Payal Bhardwaj, Department of Biophysics, Panjab University, Basic Medical Sciences Block, Chandigarh, 160014, India, E-mail: payalpu_82@yahoo.co.in

Received April 13, 2016; Accepted April 27, 2016; Published April 28, 2016

Citation: Bhardwaj P, Rai D, Garg M (2016) Zinc Improves the Bone Mechanical Strength in Ovariectomized Rat Model by Restoring Bone Composition and Hydroxyapatite Crystallite Dimension. Vitam Miner 5: 137. doi:10.4172/23761318.1000137

Copyright: @ 2016 Bhardwaj $P$, et al. This is an open-access article distributed under the terms of the Creative Commons Attribution License, which permits unrestricted use, distribution, and reproduction in any medium, provided the original author and source are credited. 
zinc-related proteins are known to play a role in regulation of cellular activity of both the osteoclast and osteoblast cells. Zinc deficiency is associated with many kinds of skeletal disorders. Some studies have also shown that osteoporotic patients have lower levels of skeletal zinc as compared to the healthy person. Also, in postmenopausal women, urinary zinc has been suggested as a marker of bone resorption [13]. Furthermore, in the previous research study from our laboratory, we have found decreased zinc concentrations in both the femur and tibia bone as a result of estrogen deficiency [14]. Zinc supplementation in such condition showed normalization of antioxidant defense system, bone tissue zinc levels and restoration of cortical bone micro architecture. Trabecular bone architecture could also be restored upon zinc administration as shown in one of our study [15]. From these investigations, we came to the conclusion that zinc supplementation in the initial stages of bone loss could somehow arrest the bone tissue deterioration. However, the action of zinc supplementation on the nanostructure scale and the ultimate mechanical strength is yet to be elucidated. Therefore, the current study has been conducted with the intention to get information pertaining to the crystallite size of mineral present in bone, the chemical compositional changes and the mechanical strength following ovariectomy and zinc supplementation.

The oxidative defense system is one of the major pathways for bone loss due to estrogen deficiency is the oxidative defense system. It has been very well demonstrated that reactive oxygen species (ROS) generation in bone tissues plays a key role in the modulation of bone cell function and influences the pathophysiology of mineralized tissues [16]. Postmenopausal condition causes increased ROS. This increase stimulates osteoclastogenesis through the RANKL (receptor activator for nuclear factor $\mathrm{kB}$ ligand) pathway in addition to the apoptosis of osteoblasts. Both these processes will ultimately cause bone loss [17]. Zinc directly improves the antioxidant defense system of bone by recovering estrogen levels [14].

To carry out the above mentioned investigations, rats that are reproductively mature and capable of responding appropriately to estrogen deficiency and its consequences after ovariectomy were taken. By performing ovariectomy at 12 weeks and keeping a treatment period of 8 weeks, osteopenic rat model was developed. The effects of zinc supplementation on bone loss prevention post ovariectomy were studied by assessing bone mechanical strength, bone matrix analysis and hydroxyapatite crystallite size.

\section{Materials and Methods}

The present study has been carried out using forty eight female Wistar rats (12 weeks old, 100-150 g) procured from the Animal House of Panjab University central facility, Chandigarh. The animals were given clean drinking tap water and standard animal pellet diet (Ashirwad Industries, Kharar, Punjab, India), throughout the experiment ad libitum. The animals were randomly segregated into four groups consisting of twelve animals in each group. The animals taken were acclimatized for a period of one week to laboratory conditions before the start of the experimental procedure. Group 1 (Control) animals were given standard diet as well as drinking water. Group 2 (Zinc) animals were given zinc supplementation as zinc sulphate in drinking water at a dose level of $227 \mathrm{mg}$ of $\mathrm{ZnSO}_{4} .7 \mathrm{H}_{2} \mathrm{O}$ per liter [18]. Group 3 (OVX) animals were ovariectomized and were kept under normal laboratory feeding conditions. Group 4 (OVX+Zinc) animals were bilaterally ovariectomized and were given zinc in drinking water at a similar dose level to that of group 2 animals. The treatment period was continued for eight weeks. The experimental design and procedures were approved by the Ethical Committee on Animal Experiments of the Central Animal House, Panjab University, Chandigarh (UT), India.

\section{Surgical procedure for bilateral ovariectomy}

Ovariectomy was performed in female Wistar rats by the method as described in our previous paper [14]. The ovariectomized Wistar rat model has been recommended by Food and Drug Administration (FDA) as the best model applied for the rapid development of osteoporosis for use in pathogenesis and treatment of bone loss [19].

After the completion of eight weeks, blood was collected from all the treatment groups by puncturing retro orbital plexus. Blood samples were then centrifuged at $1,500 \mathrm{rpm}$ to separate serum. Thereafter, the animals were sacrificed by decapitation under anesthesia and both the bones i.e., femora and tibiae were extracted from the animals of all treatment groups. Bones taken out from the animals were cleared off from the muscle and cartilage tissue and were then processed further.

\section{Bone resorption markers}

Estimation of serum estradiol levels: Measurement of estradiol levels reveals the efficacy of the bilateral ovariectomy surgery protocol. Estradiol levels in the collected serum were measured using Kit from DRG Diagnostics GmbH, Germany, by spectrophotometric method. Principle of this method is based on the competitive binding of horse radish peroxidase estradiol with the estradiol in the sample. The detail procedure has been mentioned in our previous publication [14].

Serum tartarate resistant acid phosphatase (TRAP-5b) levels: Serum TRAP-5b secreted by osteoclasts is a marker of bone resorption. TRAP-5b was measured spectrophotometrically using TRAP kit from Immuno Diagnostics System UK. It is a solid phase immunofixed enzyme activity assay for the determination of osteoclast derived TRAP-5b form in rat serum. TRAP is a marker for osteoclast cells (bone resorption marker). In this test, IgG-coated microtiter wells incubated with monoclonal antibody were used. After washing with phosphate buffer saline, standard, control and serum samples were incubated in the wells, and bound TRAP-5b activity was determined with a chromogenic substrate to develop a color. The reaction was stopped and the absorbance of reaction mixture was read at $405 \mathrm{~nm}$ [20].

Alkaline phosphatase (ALP): Alkaline phosphate is secreted by osteoblast cells and therefore is a marker of bone formation. ALP activity in the serum was determined by the method of Walter and Schutt [21]. Reaction mixture consisting of $900 \mu \mathrm{l}$ of p-nitro phenyl phosphate and $1.3 \mathrm{ml}$ of glycine buffer was incubated for $5 \mathrm{~min}$ at $37^{\circ} \mathrm{C}$. Then the serum sample $(200 \mu \mathrm{l})$ was added to the reaction mixture which was again allowed to incubate for about 30-35 min. The final reaction was stopped by the addition of $0.1 \mathrm{~N}$ Sodium hydroxide. Alkaline Phosphatase present in the sample catalyzes the conversion of p-nitrophenyl phosphate (PNPP) to p-nitrophenol. P-nitrophenol is a bright yellow-colored compound which has maximum absorbance at $405 \mathrm{~nm}$. The rate of increase in absorbance from P-nitrophenyl phosphate (colorless) to P-nitrophenol (color) is directly proportional to the alkaline phosphatase enzyme activity in the sample. Enzyme activity was expressed as $\mu \mathrm{mol}$ of $\mathrm{p}$-nitrophenol liberated per min per mg protein.

\section{Bone matrix analysis using Fourier Transformer Infrared Spectroscopy (FTIR)}

Fourier transformer infrared spectroscopy provides a powerful way to study the structure and nature of interaction of inorganic and organic matrix and the changes which are associated with many pathological conditions of bone. For sample preparation, the bones (both femur and tibia) were allowed to dry at $90^{\circ} \mathrm{C}$ in the incubator for about $48 \mathrm{hrs}$. Then the dried bone specimen were crushed into 
powder form with the help of an agate pestle and mortar and sieved through the 80 micron sieve to get the fine powder for analysis. The powdered samples of all the groups were then mixed with Potassium Bromide (KBr, Spectroscopic grade) in the ratio of 5:95 (Sample: $\mathrm{KBr}$ ). The mixture was then made into circular pellets of around $1.5 \mathrm{~cm}$ diameter at a pressure of $10^{4}$ Newtons using hydraulic press (Perkin Elmer). Pellets were then kept in the sample holder of the FTIR and the spectra were recorded in the range of $4000-400 \mathrm{~cm}^{-1}$ by using FTIR (RZX, Perkin Elmer) in the Sophisticated Analytical Instrumentation Facility, Panjab University, Chandigarh, India.

\section{X-Ray diffraction studies}

$\mathrm{X}$-ray diffraction analysis was done to obtain information about the crystallanity changes in the bone mineral after ovariectomy and upon zinc supplementation.

The bone powder was prepared in the similar manner as for the FTIR analysis. For the powder X-ray analysis, Panalytical's XPert Pro X-ray Diffractometer with Goniometer (PW3050/60) was used. The bone powders were packed tightly in the rectangular aluminium cell and exposed to the X-ray beam from X-ray generator running at 45 $\mathrm{KV}$ and $40 \mathrm{~mA}$. The scanning regions of the diffraction angles $2 \theta$ were 5 to $80^{\circ}$, which covered most of the significant diffraction peaks of the bone crystallites. After the acquisition of data, the Scherrel's formula was applied to each sample for the calculation of crystallite size [22].

The average crystallite size of bone apatite was calculated on the basis of the Scherrer's formula (Patterson 1939), which is:

\section{$\mathrm{L}=K \lambda / \beta \cos \theta$}

$\mathrm{L}$ : the average crystallite size; $\mathrm{K}$ : is a constant related to crystallite shape, normally taken as $0.9 ; \theta$ : the angle of reflection belonging to the main point of the curve; $\lambda: 1.54 \mathrm{~A}^{\circ}$ wavelength of $\mathrm{Cu}$ Ka radiation; $\beta$ : FWHM is the width of the diffraction peak profile at half maximum height.

\section{Bone mechanical strength}

The mechanical properties of the bone were measured with the help of texture analyzer using three point bending ridge probes (Model TA-XT2i, Stable Micro Systems) from Punjab Biotechnology Incubator, Mohali. A three-point bending device is adapted for the small dimensions of the bones and consisted of two beams spaced a known distance apart, supporting the lower bone. Bone samples were prepared by flushing out the bone marrow from the excised bone tissues (both femur and tibia) after giving a cut on diaphyseal ends of the bone. The bone samples were then kept in normal saline for the period of $24 \mathrm{hrs}$. for further use.

\section{Statistical analysis}

All the data obtained in the current study were expressed as the mean \pm the standard deviation (SD). One-way analysis of variance has been applied to evaluate differences among groups. NewmanKeuls post hoc test was applied multiple comparisons between groups. Differences between various groups were considered statistically significant at $\mathrm{P}<0.05$. The data processing was done using statistical analysis software SPSS (version 14).

\section{Results}

\section{Markers of bone resorption}

Serum estradiol levels were presented in Table 1 , which is a measure of the efficacy of surgery protocol. The data showed significant decrease in the estradiol levels in OVX group $(\mathrm{p}<0.001)$ when compared with the control. Zinc administration has caused significant $(\mathrm{p}<0.001)$ improvement in these levels. Also, serum TRAP-5b activity in the OVX group was found to be significantly higher $(\mathrm{p}<0.001)$ compared to the control. The values were restored to normal upon zinc administration. Ovariectomized group showed increased serum alkaline phosphatase activity $(\mathrm{p}<0.001)$ as compared to the control group, which got reduced to normal values $(\mathrm{p}<0.001)$ upon zinc supplementation.

\section{Assessment of bone mineral and matrix components by using FTIR}

Figure 1 represents the typical spectra of the bone tissue showing prominent vibrational bands related to the bone tissue composition. The band centered at $1028 \mathrm{~cm}^{-1}$ corresponds to the symmetric stretching vibration $\left(\mathrm{v}_{1}\right)$ of phosphate $\left(\mathrm{PO}_{4}^{3-}\right)$ and is the strongest marker of bone mineral. The band assigned between $905 \mathrm{~cm}^{-1}$ to $825 \mathrm{~cm}^{-1}$ corresponds to the carbonate $\left(\mathrm{CO}_{3}^{2-}\right)$ vibrational mode and indicated the extent of carbonate incorporated into the hydroxyapatite lattice. The peaks for the collagen matrix are the amide III (around $1451 \mathrm{~cm}^{-1}$ ) and amide I (around $1660 \mathrm{~cm}^{-1}$ ) which arises due to the collagen present in the bone. In the current study, amide 1 peak was chosen specifically, that corresponds to the type 1 collagen, the most abundant type present in the bone tissue [23]. The areas under the bone specific peaks were calculated and tabulated in Table 2 .

Hydroxyl peak femur (3000-4000 $\left.\mathrm{cm}^{-1}\right)$ : The results indicated no significant alteration in the area under the hydroxyl peak (3000-4000 $\mathrm{cm}^{-1}$ ) of the femur of OVX group in comparison to the control group. However, significant reduction $(\mathrm{p}<0.01)$ of the area under the hydroxyl peak of OVX tibia was observed. Significant increment $(p<0.001)$ in the peak area was observed in the femur of OVX+Zinc group in comparison to the OVX group but increase was not significant for tibia bone. Zinc supplementation to the control animals also showed increase in the hydroxyl peak area $(p<0.05)$ for femur and showed no alteration in the tibia bone peak area.

Amide peak femur (1574 to $\left.1729 \mathrm{~cm}^{-1}\right)$ : The data indicated significant reduction in the area under amide peak for both femur and tibia bone of the OVX group as compared to the control group. Zinc supplementation to the OVX group showed significant $(\mathrm{p}<0.001)$ increase in the amide peak area of femur bone when compared to the OVX group and also in comparison to the control group $(\mathrm{p}<0.001)$. Zinc supplementation to the OVX group showed no change in the peak area of the tibia bone. However, the amide peak area remained almost same when zinc was supplemented to the normal control animals for both the femur as well as tibia.

Phosphate femur (930 to $1220 \mathrm{~cm}^{-1}$ ): OVX group showed significant decrease $(\mathrm{p}<0.05)$ in the area of the phosphate peak of both the femur as well as tibia bone when compared to the control group. Zinc supplementation to the OVX animals increased the peak

\begin{tabular}{|c|c|c|c|}
\hline Groups & Estradiol (pg/ml) & $\begin{array}{c}\text { TRAP-5b (serum) } \\
\text { U/L }\end{array}$ & $\begin{array}{c}\text { Alkaline } \\
\text { Phospahatse } \\
\text { (serum) U/L }\end{array}$ \\
\hline Control & $9.30 \pm 0.62$ & $3.1 \pm 0.4$ & $59 \pm 5$ \\
\hline Zinc & $8.96 \pm 0.84$ & $3.3 \pm 0.5$ & $58 \pm 7$ \\
\hline Ovx & $5.75 \pm 0.50^{\mathrm{c}}$ & $7.2 \pm 1.0^{\mathrm{c}}$ & $77 \pm 4^{\mathrm{c}}$ \\
\hline Ovx+Zinc & $7.23 \pm 0.7^{\mathrm{f}}$ & $3.6 \pm 0.7^{\mathrm{e}}$ & $62 \pm 7^{\mathrm{f}}$ \\
\hline
\end{tabular}

Each value is the mean $\pm S D(n=6)$.Values with a superscript are significantly different from the control group $\left({ }^{a}, p<0.05 ;{ }^{b}, p<0.01 ;{ }^{c}, p<0.001\right)$ or from Ovx group $\left({ }^{d}, p<0.05 ;{ }^{e}, p<0.01 ;{ }^{f}, p<0.001\right)$

Table 1: Effect of OVX and Zinc supplementation on the serum estradiol, TRAP-5b levels and alkaline phosphatase activity. 


\section{Control}

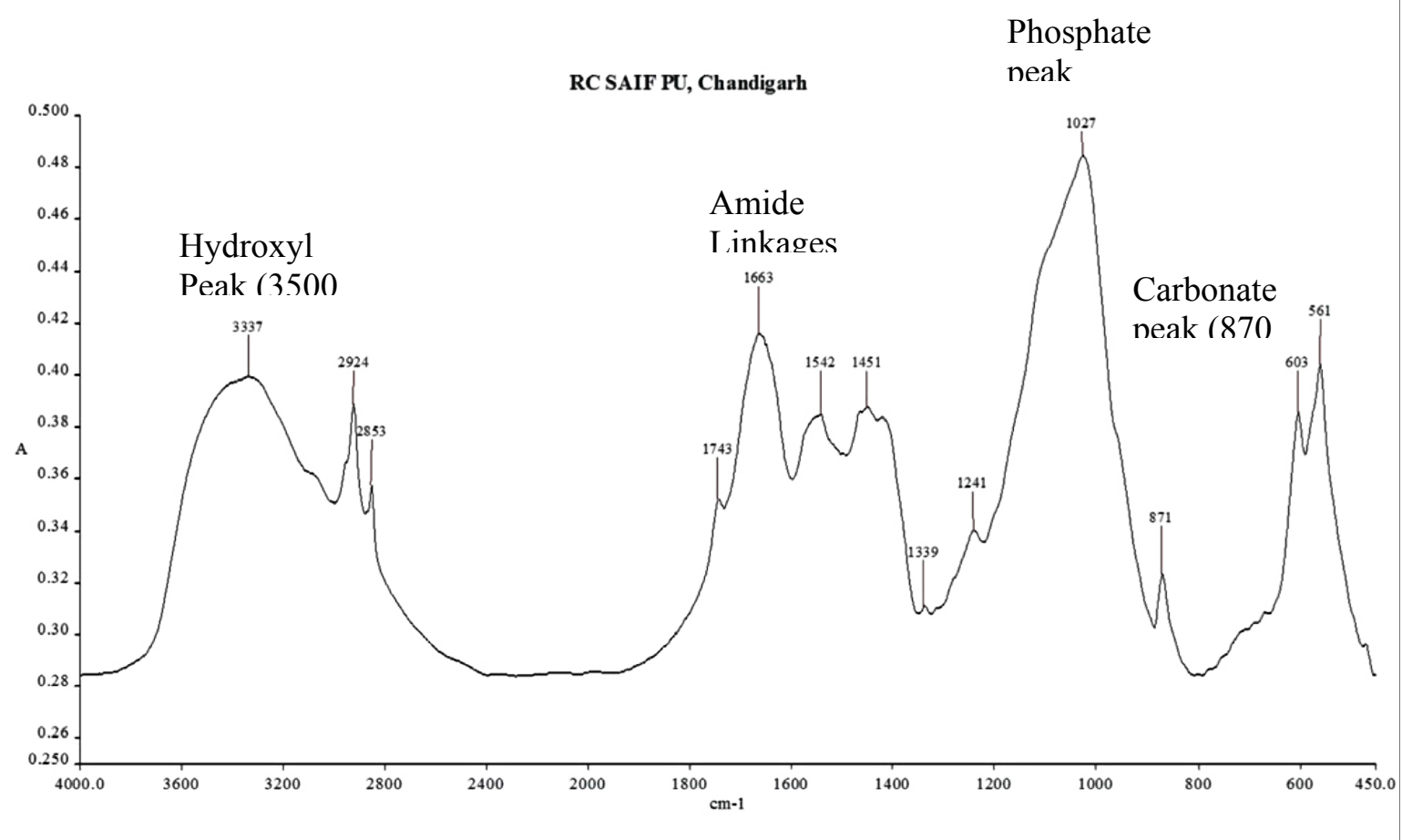

Figure 1: Typical infrared spectrum of the bone tissue obtained from the control group showing the major peaks of the molecular constituents.

\begin{tabular}{|c|c|c|c|c|c|c|c|c|}
\hline \multirow[t]{2}{*}{ Groups } & \multicolumn{2}{|c|}{$\begin{array}{l}\text { Area under the hydroxyl peak } \\
\left(3000 \text { to } 4000 \mathrm{~cm}^{1}\right)\end{array}$} & \multicolumn{2}{|c|}{$\begin{array}{l}\text { Area under the amide peak } \\
\left(1574 \text { to } 1729 \mathrm{~cm}^{1}\right)\end{array}$} & \multicolumn{2}{|c|}{$\begin{array}{l}\text { Area under the phosphate peak } \\
\left(930 \text { to } 1220 \mathrm{~cm}^{-1}\right)\end{array}$} & \multicolumn{2}{|c|}{$\begin{array}{l}\text { Area under the carbonate peak } \\
\left(905 \text { to } 825 \mathrm{~cm}^{-1}\right)\end{array}$} \\
\hline & Femur & Tibia & Femur & Tibia & Femur & Tibia & Femur & Tibia \\
\hline Control & $38 \pm 7$ & $43 \pm 16$ & $4.5 \pm 0.7$ & $4.1 \pm 1.8$ & $30 \pm 5$ & $30 \pm 12$ & $0.47 \pm 0.11$ & $0.58 \pm 0.23$ \\
\hline Zinc & $57 \pm 16^{a}$ & $39 \pm 8$ & $5.5 \pm 1.5$ & $3.6 \pm 1.1$ & $41 \pm 13$ & $29 \pm 11$ & $0.87 \pm 0.26^{b}$ & $0.37 \pm 0.08$ \\
\hline OVX & $33 \pm 5$ & $19 \pm 9^{b}$ & $3.3 \pm 0.2$ & $2.1 \pm 0.9^{a}$ & $19 \pm 2^{a}$ & $13 \pm 4^{a}$ & $0.26 \pm 0.04$ & $0.21 \pm 0.08^{b}$ \\
\hline OVX+Zinc & $71 \pm 13^{f, c}$ & $30 \pm 9$ & $7.9 \pm 1.8^{f, c}$ & $2.4 \pm 0.3$ & $50 \pm 9^{f, b}$ & $21 \pm 5$ & $1.05 \pm 0.19^{f, c}$ & $0.44 \pm 0.19^{d}$ \\
\hline
\end{tabular}

Each value is the mean $\pm \mathrm{SD}(n=5)$. Values with a superscript are significantly different from the control group $\left({ }^{a}, p<0.05 ;{ }^{b}, p<0.01 ;{ }^{c}, p<0.001\right)$ or from Ovx group $\left({ }^{d}\right.$, $\left.p<0.05 ;{ }^{e}, p,<0.01 ;{ }^{f}, p<0.001\right)$

Table 2: Area under the bone mineral and organic bands of femur and tibia obtained from all the four groups.

area of femur bone $(\mathrm{p}<0.001)$ when compared to the OVX group but the increase was not found to be significant for tibia bone. Zinc supplementation alone to the control animals also showed elevation in the peak area but the increase was not significant in comparison to the control group.

Carbonate femur (905 to $\left.825 \mathrm{~cm}^{-1}\right)$ : Carbonate peak area of the ovariectomized group was found to decrease for femur and tibia bone $(\mathrm{p}<0.01)$ but the decrease does not differ significantly in the case of femur bone. The area of this peak was significantly increased for both femur $(\mathrm{p}<0.001)$ and tibia bone $(\mathrm{p}<0.05)$ when zinc was supplemented to the ovariectomized animals. Zinc supplementation alone to the control animals also showed significant increase $(\mathrm{p}<0.01)$ in the carbonate peak area of femur when compared to the control group. However, zinc supplementation alone to the control animals showed no significant changes in the peak area of tibia bone.

\section{$\mathrm{X}$-ray Diffraction (XRD) studies}

Figures 2 and 3 represent the diffraction pattern of the femur and tibia bone tissue respectively. The main peaks are from the planes of (200), (111), (002), (102), (210), (211), (112), (300), (202), (212), (310),
(311), (113), (222), (312) and (213) of hydroxyapatite phase for $20^{\circ}$ $50^{\circ} 2 \theta$ of the JCPDS: 9-432 standard (Monshi et al.). As seen from the $\mathrm{X}$-ray diffraction spectra (Figures 2 and 3 ), the peak at around $2 \theta=32.3^{\circ}$ corresponding to hydroxyapatite $\left[\mathrm{Ca}_{10}\left(\mathrm{PO}_{4}\right)_{6}(\mathrm{OH})_{2}\right]$ was observed in all the groups. One more peak was observed at around $2 \theta=25.8^{\circ}$.

The crystallite size of the hydroxyapatite crystal of both the bone tissues was calculated using Scherrer's formula applying FWHM ( $\beta$ ) for (002) and (211) planes and the results are tabulated in Table 3. Enhancement in the crystallite size of the hydroxyapatite crystal of ovariectomized rat bone was found as is evident from the Table 3. Zinc supplementation to the ovariectomized animals causes reduction in the crystallite size when compared to the OVX group.

\section{Bone mechanical strength}

Breaking strength of the femur as well as tibia was found to decrease significantly $(\mathrm{p}<0.001)$ as compared to the control group which increased significantly upon zinc supplementation (Table 4). However, zinc treatment alone to the control animals showed no significant alteration in the breaking strength values when compared to the control group. 

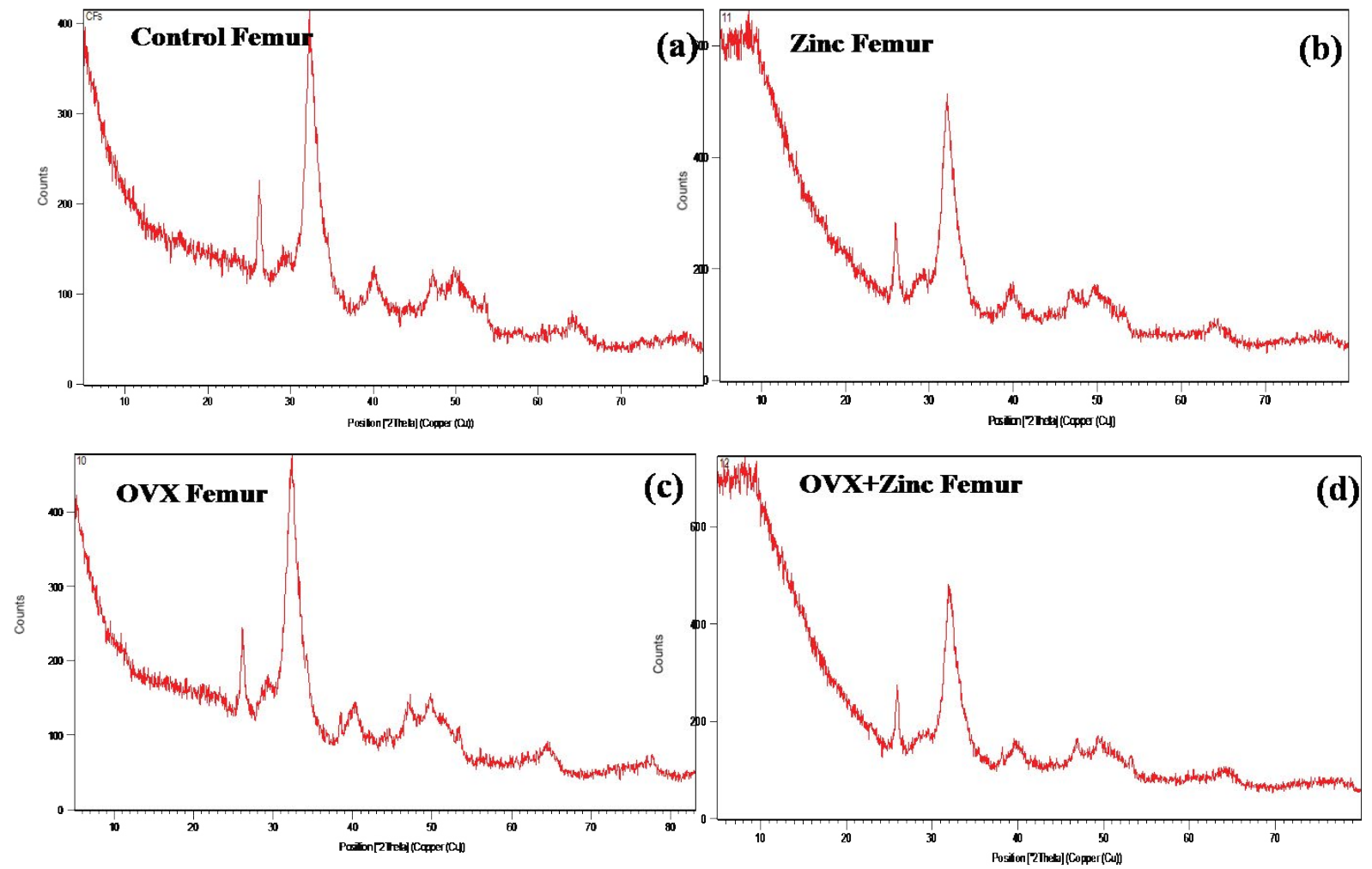

Figure 2: X-ray diffraction pattern of the femur bone tissue obtained from all the treatment groups.
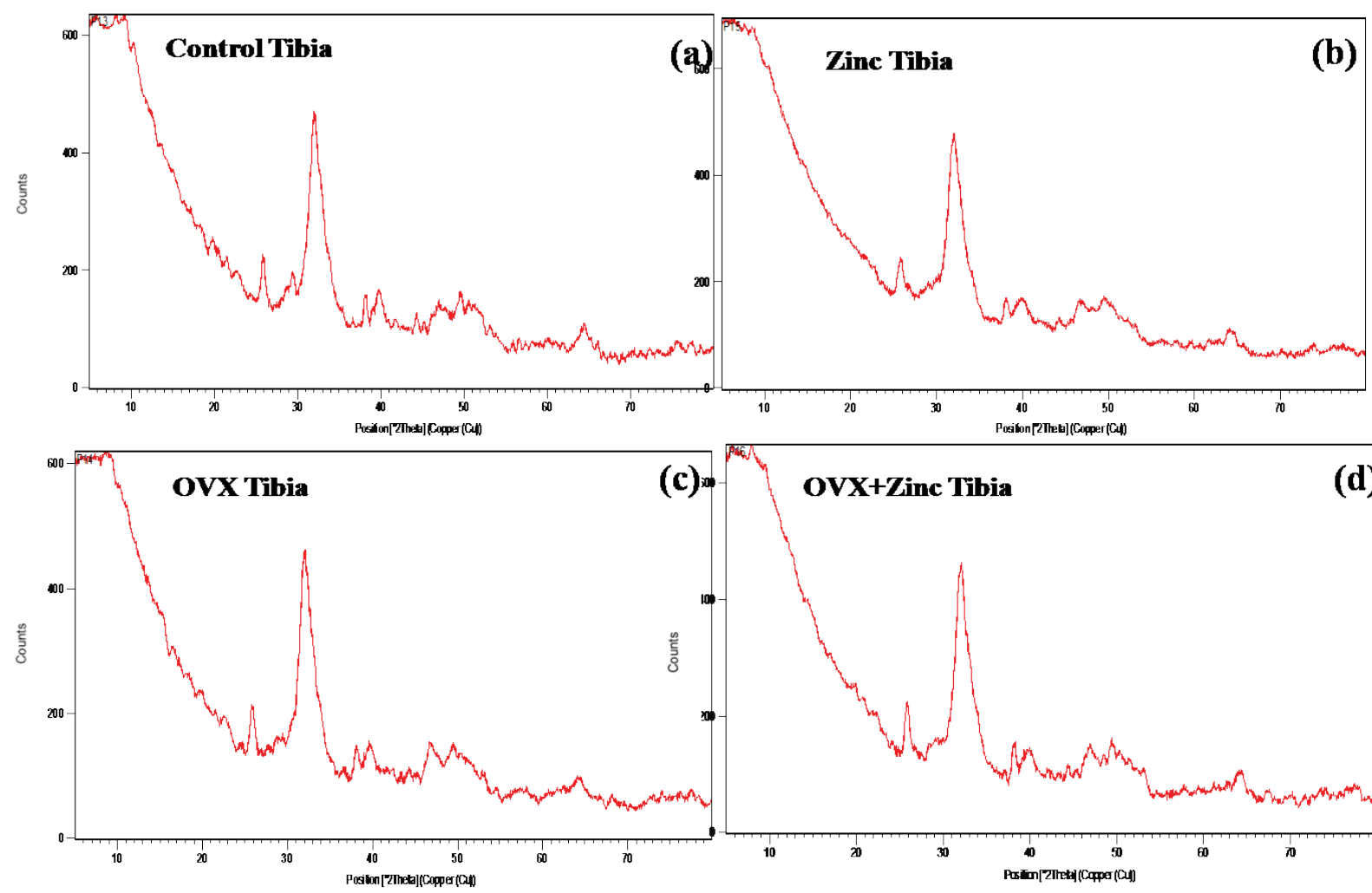

(c)

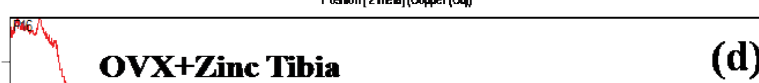

Figure 3: X-ray diffraction pattern of the tibia bone tissue obtained from all the treatment groups. 


\begin{tabular}{|c|c|c|c|c|c|c|c|c|c|c|}
\hline \multirow[t]{2}{*}{ Groups } & \multicolumn{2}{|c|}{ Peak (002) } & \multicolumn{2}{|c|}{ FWHM } & \multicolumn{2}{|c|}{ Peak (211) } & \multicolumn{2}{|c|}{ FWHM } & \multicolumn{2}{|c|}{$\begin{array}{l}\text { Average hydroxyapatite } \\
\text { crystallite size (nm) }\end{array}$} \\
\hline & Femur & Tibia & Femur & Tibia & Femur & Tibia & Femur & Tibia & Femur & Tibia \\
\hline Control & 26.27 & 25.98 & 0.40 & 0.33 & 32.28 & 31.99 & 0.49 & 0.27 & 24 & 26 \\
\hline Zinc & 25.98 & 26.00 & 0.40 & 0.30 & 32.07 & 32.09 & 0.33 & 1.51 & 21 & 29 \\
\hline ovX & 25.91 & 25.83 & 0.17 & 0.30 & 31.94 & 32.06 & 0.47 & 0.15 & 31 & 38 \\
\hline OVX+Zinc & 26.11 & 25.87 & 0.40 & 0.47 & 34.33 & 36.64 & 0.30 & 0.60 & 22 & 15 \\
\hline
\end{tabular}

Table 3: Average crystallite size of the femur and tibia bone.

\begin{tabular}{|c|c|c|}
\hline & \multicolumn{2}{|c|}{ Breaking force (N) } \\
\hline Groups & Femur & Tibia \\
\hline Control & $95 \pm 2$ & $58.0 \pm 0.3$ \\
\hline Zinc & $93 \pm 1$ & $57.4 \pm 2.9$ \\
\hline Ovx & $68.6 \pm 0.8^{\mathrm{c}}$ & $45 \pm 1^{\mathrm{c}}$ \\
\hline Ovx+Zinc & $87.7 \pm 0.9^{\mathrm{f}}$ & $52 \pm 2^{\mathrm{e}}$ \\
\hline
\end{tabular}

Each value is the mean $\pm S D(n=4)$.Values with a superscript are significantly different from the control group ( $\left.{ }^{a}, p<0.05 ;{ }^{b}, p<0.01 ;^{c}, p<0.001\right)$ or from Ovx group ( $\left.{ }^{d}, p<0.05 ;{ }^{e}, p<0.01 ;{ }^{f}, p<0.001\right)$

Table 4: Mechanical strength analysis of femur and tibia bone.

\section{Discussion}

The current study showed that zinc supplementation in an ovariectomized osteopenic rat model improved the bone mechanical strength by restoring the bone material properties and hydroxyapatite crystallite size.

\section{Bone resorption markers}

Glands of the female reproductive system gradually reduce their production of female sex hormones, resulting in cessation of menstruation during post-menopausal condition. As a result of it, number of biological processes gets adversely affected [24]. Ovariectomized Wistar rat model was used for the present investigations which have been approved by FDA [25]. Removal of the ovaries has also been shown to be associated with increased risk of death from heart disease [26].

The estradiol levels were evaluated using competitive binding assay for the confirmation of surgery protocol. The results showed significant decrease in the estradiol levels following ovariectomy which gives the confirmation of the success of the surgery protocol (Table 1). The decline in the estradiol levels following ovariectomy might have up regulated the formation of number of cytokines and further differentiation and maturation of osteoclast cells. This will cause more secretion of RANKL released from the osteoblast cells apart from osteoprotegrin (OPG) protein, which binds to the RANKL and prevents it from activating RANK receptor. This whole cascade will stimulate osteoclastogenesis and ultimately bone loss [27-29]. Zinc supplementation to the ovariectomized animals has caused increment in the estrogen levels suggesting the potential role of zinc in the secretion of estrogen from glands other than ovary [14].

TRAP-5b activity, a direct indicator of osteoclastogenesis [30] and a marker of bone resorption was assessed in serum. Increased TRAP-5b levels in the OVX group (Table 1) give us the confirmation of increased bone resorption following estrogen deficiency. These findings are in agreement with the previous studies reporting greater concentrations of TRAP-5b in the serum of the OVX group [31]. The decreased TRAP-5b levels observed after zinc supplementation in the OVX group substantiates that zinc is a potent factor in bone remodeling by inhibiting bone resorption.

The increased TRAP-5b levels following ovariectomy might have caused collagen fragmentation as earlier studied by Halleen group
[32]. The increase in the serum alkaline phosphatase activity in the ovariectomized animals was observed which was earlier described by Kalu et al. [19]. These increased levels were restored to normal upon zinc supplementation.

The above mentioned metabolism markers suggested that, in the ovariectomized group, estrogen levels decreased significantly, serum alkaline phosphatase and TRAP-5b levels increased following ovariectomy. These observations confirm that the ovariectomized model has been established, in which the bone resorption has enhanced and bone loss has appeared. Also, upon zinc supplementation, these bone metabolism markers get modulated showing the effective role of zinc supplementation in the regulation of bone metabolism. Further the biophysical techniques were applied so as to characterize the material properties of bone and also to evaluate the bone mechanical strength following ovariectomy and zinc supplementation.

\section{Analysis of various bone components using Fourier Transformer Infra-Red Spectroscopy (FTIR)}

In the present work, FTIR spectra obtained for all the groups were dominated by a very strong band around $1028 \mathrm{~cm}^{-1}$ assigned to the symmetrical stretching mode of the phosphate group. The variations in the intensity of the $\mathrm{PO}_{4}^{3-}$ peak indicate different degree of mineralization. Carbonate band was present near $871 \mathrm{~cm}^{-1}$ which reveals the extent of carbonate substitution in the hydroxyapatite mineral crystal. The peaks for the collagen matrix are the amide III $\left(1451 \mathrm{~cm}^{-1}\right)$ and amide I (1665 $\mathrm{cm}^{-1}$ ) which arises due to the collagen protein present in the bone. For the comparison of the data, the areas under the observed peaks representing different molecules were calculated and are presented in Table 2 .

There was significant decrease in the area under the phosphate peak of both the bones, which is a measure of mineralization. This showed that the mineralization process gets affected following ovariectomy. However, zinc supplementation to the OVX animals increased the phosphate content of the bones showing the potential role of zinc in the enhancement of mineralization process. It has also been observed from the data that zinc also enhanced mineralization when supplemented to the normal control animals. We have taken sexually mature growing rats in which bone tissue mineralization process is still in process and thus, zinc administration to the normal control animals appears to cause further increment in the normally ongoing mineralization process.

A decrease in the total carbonate content of the osteopenic bone (Table 2) in comparison to the control bone was observed in the present study. This result is consistent with the earlier result obtained in the ovariectomized model [33]. The alteration in the carbonate content reflects the amount of carbonate substitution in the bone mineral environment. The decrease in the carbonate content following ovariectomy can be attributed to the selective resorption of the more mature bone which is towards the endocortical portion. The effect may also be due in part to the change in carbonate content of the extracellular fluids and, therefore, in the uptake of carbonate by bone mineral 
component. The area under this peak was increased significantly when zinc was administered to the ovariectomized animals. This shows that ingestion of zinc had pronounced effects on the bone formation process thereby leading to the formation of more mature bone. However, zinc supplementation alone to the control animals showed no significant changes in the peak area in case of tibia whereas there was a significant increase in the case of femur.

The band around $1660 \mathrm{~cm}^{-1}$ was a composite of amide groups and water molecules. From the Table 2, it is evident that there is significant reduction in the peak area of amide linkages in the ovariectomized animals as compared to the control animals. The reduction in the amide peak could be due to the reduction in the amide linkages as a result of collagen fragmentation. Ovariectomized condition might have resulted in collagen fragmentation due to increase in TRAP-5b levels. Increased TRAP-5b levels in the osteopenic condition were also observed in the previous study conducted in our laboratory [14]. Zinc supplementation to the OVX group showed no change in the peak area when compared to the OVX group. Also, zinc supplementation to the normal control animals showed no significant change in the peak area when compared to the control group.

An important observation from the FTIR results is that in the case of OVX tibia, there was significant reduction observed in all the peak areas specific for hydroxyl, amide, phosphate and carbonate contents (Table 2). However, in the case of OVX femur bone, reduction in the peak area was found to be significant only for the phosphate peak. From this it can be said that the resorption following estrogen deficiency was more pronounced in the case of tibia when compared to the femur bone. Earlier study also showed similar results, where enhanced bone resorption rate in tibia, a load bearing bone, in comparison to the femur bone in the OVX condition was observed [34]. Thus the regulation of bone turnover by zinc supplementation has prevented the deterioration of both the bones by restoring the altered chemical composition.

\section{X-ray diffraction studies}

X-ray diffractions studies indicated increased crystallite size of the hydroxyapatite mineral of ovariectomized rat bone as is evident from the Table 3. Similar results were shown by various authors demonstrating that osteoporotic bone displays larger crystal $[35,36]$. Increased bone hydroxyapatite particle size is linked with increased bone fragility; crystals that are too small do not reinforce the bone composite material, suggesting that there is also an optimal size range for bone mineral crystals. Larger crystal will tend to reduce the stiffness of the bone and make it more fragile. Zinc supplementation to the OVX animals restores the altered crystallite size as a result of estrogen deficiency. As the analysis was carried out using only four samples (two femur and two tibiae) from each treatment groups for estimating the crystallite size, which may not be sufficient, therefore, further additional studies can be carried out on large number of samples so as to get more in depth information on hydroxyapatite crystal. However, in the current study, we have validated our data with various other sophisticated techniques also, which has distinctly defined the protective potential of zinc in arresting early stages of bone loss.

\section{Bone mechanical strength}

Estrogen deficient condition in rats induces changes in bone mechanical properties (Table 4) which get modulated upon zinc supplementation. Estrogen deficiency is well known to derange the bone metabolism and causes osteoporosis. This actually impairs the osteoblastic activity that results in reduced bone formation and mineralization. Loss of mineral is thus a major cause of increase susceptibility to bone fracture. We have observed a significant decrease in the breaking strength of the bones (both femur and tibia) of the OVX group which is attributed to the decreased bone matrix components (Table 2), increased hydroxyapatite crystallite size (Table 3). This is in tune with the similar studies suggesting decrease in the bone strength following ovariectomy [37]. Increased bone resorption during estrogen deficiency resulted into large number of resorption pits and increased diameter of haversian canal [14]. During the mineral loss, the area which was earlier occupied by the mineral would get replaced by the water molecules from the surroundings. By this, bone volume gets preserved but its overall strength decreases. Mineral deficit in bone and enhanced bone water concentration were also studied by the Anumula group [38] applying sophisticated techniques like nuclear magnetic resonance studies and micro-computed tomography. However, Zinc supplementation to the ovariectomized animals restores the bone strength thus suggesting the major role of zinc in mineralization and collagen synthesis in early stages of bone loss.

Although immense debate still exists on the mechanism for osteoporosis and its reversal upon nutritional supplementation. These nutritional supplements impact the balance between formation and resorption processes on skeletal metabolism. Based on the results of the current work, we have concluded that supplemental intake of zinc may play preventive and therapeutic role for bone loss that are induced by postmenopausal condition. Zinc helps in maintaining the mechanical strength of the bone by restoring the bone composition and microstructure. However, further studies need to be carried out to find out the exact mechanism of zinc action on bone cells in the osteopenic condition.

\section{Acknowledgements}

Authors would like to acknowledge the financial assistance provided by Indian Council of Medical Research (ICMR, No. 3/1/2/4/10-RHN, dated $9^{\text {th }}$ Aug 2010) in terms of Senior Research Fellowship (SRF) and contingency for the acquisition of various chemicals required to conduct the current research work.

\section{References}

1. Kilbanski A, Adams-Campbell L, Bassford T, Blair SN, Boden SD, et al (2001) Osteoporosis prevention, diagnosis, and therapy. JAMA 285: 785-795.

2. Hauge EM, Steiniche T, Andreassen TT (2003) Histomorphometry of metabolic bone conditions. Handbook of Histology Methods for Bone and Cartilage, pp: 391-410.

3. lacono MV (2007) Osteoporosis: a national public health priority. J Perianesth Nurs 22: 175-180.

4. Rubin CD (2005) Emerging concepts in osteoporosis and bone strength. Curr Med Res Opin 21: 1049-1056.

5. Yeh IT (2007) Postmenopausal hormone replacement therapy: endometrial and breast effects. Adv Anat Pathol 14: 17-24.

6. Siris ES, Selby PL, Saag KG, Borgström F, Herings RM, et al. (2009) Impact of osteoporosis treatment adherence on fracture rates in North America and Europe. Am J Med 122: S3-13.

7. Riggs BL, Khosla S, Melton LJ 3rd (2002) Sex steroids and the construction and conservation of the adult skeleton. Endocr Rev 23: 279-302.

8. Takada Y, Aoe S, Kumegawa M (1996) Whey protein stimulated the proliferation and differentiation of osteoblastic MC3T3-E1 cells. Biochem Biophys Res Commun 223: 445-449.

9. Ammann P (2009) Bone strength and ultrastructure. Osteoporos Int 20: 1081 1083.

10. Hashizume M, Yamaguchi M (1993) Stimulatory effect of beta-alanyl-Lhistidinato zinc on cell proliferation is dependent on protein synthesis in osteoblastic MC3T3-E1 cells. Mol Cell Biochem 122: 59-64.

11. Yamaguchi M, Segawa Y, Shimokawa N, Tsuzuike N, Tagashira E (1992) Inhibitory effect of beta-alanyl-L-histidinato zinc on bone resorption in tissue culture. Pharmacology 45: 292-300. 
Citation: Bhardwaj P, Rai D, Garg M (2016) Zinc Improves the Bone Mechanical Strength in Ovariectomized Rat Model by Restoring Bone Composition and Hydroxyapatite Crystallite Dimension. Vitam Miner 5: 137. doi:10.4172/2376-1318.1000137

12. Yamaguchi M (2013) Osteoporosis treatment with new osteogenic factors. J Mol Genet Med 7.

13. Herzberg M, Foldes J, Steinberg R, Menczel J (1990) Zinc excretion in osteoporotic women. J Bone Miner Res 5: 251-257

14. Bhardwaj P, Rai DV, Garg ML (2013) Zinc as a nutritional approach to bone loss prevention in an ovariectomized rat model. Menopause 20: 1184-1193.

15. Bhardwaj P, Rai DV, Garg ML (2016) Zinc inhibits ovariectomy induced microarchitectural changes in the bone tissue Journal of Nutrition \& Intermediary Metabolism 3: 33-40.

16. Wauquier F, Leotoing L, Coxam V, Guicheux J, Wittrant Y (2009) Oxidative stress in bone remodelling and disease. Trends Mol Med 15: 468-477.

17. Bai XC, Lu D, Liu AL, Zhang ZM, Li XM, et al. (2005) Reactive oxygen species stimulates receptor activator of NF-kappaB ligand expression in osteoblast. $J$ Biol Chem 280: 17497-17506.

18. Goel A, Dhawan DK (2001) Zinc supplementation prevents liver injury in chlorpyrifos-treated rats. Biol Trace Elem Res 82: 185-200.

19. Kalu DN, Liu CC, Hardin RR, Hollis BW (1989) The aged rat model of ovarian hormone deficiency bone loss. Endocrinology 124: 7-16.

20. Goudu SNA, Rao VP, Naidu DM, Chandra RV, Jagadesh G, et al. (2010) Clinical importance of serum TRAP-5b and urine CTX-I in the assessment of bone loss in postmenopausal women. Int J Biotechnol Biochem 6: 427-434.

21. Walter K, Schuut C (1974) Methods of Enzymatic Analysis. Bermeger HV (ed.) Academic Press, New York, pp: 856-860.

22. Patterson AL (1939) The Scherrer Formula for I-Ray Particle Size Determination Physical Review 56: 978-982.

23. Paschalis EP, Mendelsohn R, Boskey AL (2011) Infrared assessment of bone quality: a review. Clin Orthop Relat Res 469: 2170-2178.

24. Persky AM, Green PS, Stubley L, Howell CO, Zaulyanov L, et al. (2000) Protective effect of estrogens against oxidative damage to heart and skeletal muscle in vivo and in vitro. Proc Soc Exp Biol Med 223: 59-66.

25. FDA (1994) Guidelines for preclinical and clinical evaluation of agents used in the prevention or treatment of postmenopausal osteoporosis. Rockville Division of Metabolism and Endocrine Drug Products, FDA.

26. Parker WH, Broder MS, Liu Z, Shoupe D, Farquhar C, et al. (2005) Ovarian conservation at the time of hysterectomy for benign disease. Obstet Gynecol 106: 219-226.
27. Bord S, Beavan S, Ireland D, Horner A, Compston JE (2001) Mechanisms by which high-dose estrogen therapy produces anabolic skeletal effects in postmenopausal women: role of locally produced growth factors. Bone 29 : 216-222.

28. Hofbauer LC, Kühne CA, Viereck V (2004) The OPG/RANKL/RANK system in metabolic bone diseases. J Musculoskelet Neuronal Interact 4: 268-275.

29. Narducci P, Bareggi R, Nicolin V (2011) Receptor Activator for Nuclear Factor kappa B Ligand (RANKL) as an osteoimmune key regulator in bone physiology and pathology. Acta Histochem 113: 73-81.

30. Kirstein B, Chambers TJ, Fuller K (2006) Secretion of tartrate-resistant acid phosphatase by osteoclasts correlates with resorptive behavior. J Cell Biochem 98: 1085-1094.

31. Henriksen K, Tanko LB, Qvist P, Delmas PD, Christiansen C, et al. (2007) Assessment of osteoclast number and function: application in the development of new and improved treatment modalities for bone diseases. Osteoporos Int 18: 681-685.

32. Halleen JM, Raisanen S, Salo JJ, Reddy SV, Roodman GD, et al. (1999) Intracellular fragmentation of bone resorption products by reactive oxygen species generated by osteoclastic tartrate-resistant acid phosphatase. J Biol Chem 274: 22907-22910.

33. Thompson DD, Posner AS, Laughlin WS, Blumenthal NC (1983) Comparison of bone apatite in osteoporotic and normal Eskimos. Calcif Tissue Int 35: 392-393.

34. Tou JC, Foley A, Yuan H, Yvonne V, Arnaud A, et al. (2008) The effect of ovariectomy combined with hindlimb unloading and reloading on the long bones of mature Sprague-Dawley rats. Menopause 2008; 15: 494-502.

35. Burnell JM, Baylink DJ, Chestnut CH 3rd, Mathews MW, Teubner EJ (1982) Bone matrix and mineral abnormalities in postmenopausal osteoporosis. Metabolism 31: 1113-1120.

36. Gadeleta SJ, Boskey AL, Paschalis E, Carlson C, Menschik F, et al. (2000) A physical, chemical, and mechanical study of lumbar vertebrae from normal, ovariectomized, and nandrolone decanoate-treated cynomolgus monkeys (Macaca fascicularis). Bone 27: 541-550.

37. Sampath TK, Simic P, Moreno S, Bukanov N, Draca N, et al. (2008) Sevelame restores bone volume and improves bone microarchitecture and strength in aged ovariectomized rats. Endocrinology 149: 6092-6102.

38. Anumula S, Magland J, Wehrli SL, Zhang H, Ong H, et al. (2006) Measurement of phosphorus content in normal and osteomalacic rabbit bone by solid-state 3D radial imaging. Magn Reson Med 56: 946-952. 